\title{
A Smart Graded-index Multimode Fiber Based Sensor Unit for Multi-parameter Sensing Applications
}

\author{
Shuo Fang, Baoyong Li, Dawei Song, Jianzhong Zhang*, Weimin Sun, Libo Yuan \\ Key Laboratory of In-Fiber Integrated Optics, Ministry of Education, Harbin 150001, China \\ Email: "zhangjianzhong@hrbeu.edu.cn
}

Received 2013

\begin{abstract}
We demonstrate a smart optical fiber sensor unit to realize a multi-parameter sensing, including temperature, curvature and strain or displacement. The sensor unit is composed of a Bragg grating in graded-index multimode fiber and a Fizeau cavity.
\end{abstract}

Keywords: Fiber Optic Sensor; Fiber Bragg Grating; Fiber Fizeau Cavity

\section{Introduction}

Fiber optic sensors offer lots of advantages including electromagnetic interference immunity, multiplexing capability of several sensors in series, and resistance to harsh environment such as high temperature and big pressure. Multi-parameter sensing is also an important trend for optical fiber sensor. Many techniques are based on multiplexing different FBGs or combining different types of sensors, such as hybrid FBG/Fizeau interferometer, hybrid FBG/long-period gratings and so on [1-3]. On the other hand, multimode fiber (MMF) based sensors [4-7] have attracted much attention recently because of their high sensitivity and low price. A step-index MMF-based temperature and strain sensor, a refractive index sensor and a curvature sensor based on MMF, and a strain and temperature sensor based on a graded-index (GI) MMF are all demonstrated. Here we propose and demonstrate a GI MMF based sensor unit to realize a multi-parameter sensing, including temperature, curvature and strain or displacement.

\section{Setup and Experiments}

The Sensor Unit is composed of several parts, shown in Figure 1. A short Bragg grating is written in a section $(\sim 3 \mathrm{~cm})$ of GI MMF with cleaved end faces and spliced with a single mode fiber. Then this structure is inserted into a micro-silicon tube of $\sim 500 \mu \mathrm{m}$ internal diameter. Another cleaved fiber of $\sim 300 \mu \mathrm{m}$ in diameter is inserted into the micro-silicon tube from the other side and a Fizeau cavity is realized by the cleaved end-faces. Ultraviolet curing glue is used to fix the positions of fibers, finally creating the sensor unit. The sensor unit is connected with a circulator. The light source is a broadband source ASE, and an OSA and personal computer (PC) based system is used to acquire and demodulate the signals from the sensor unit.

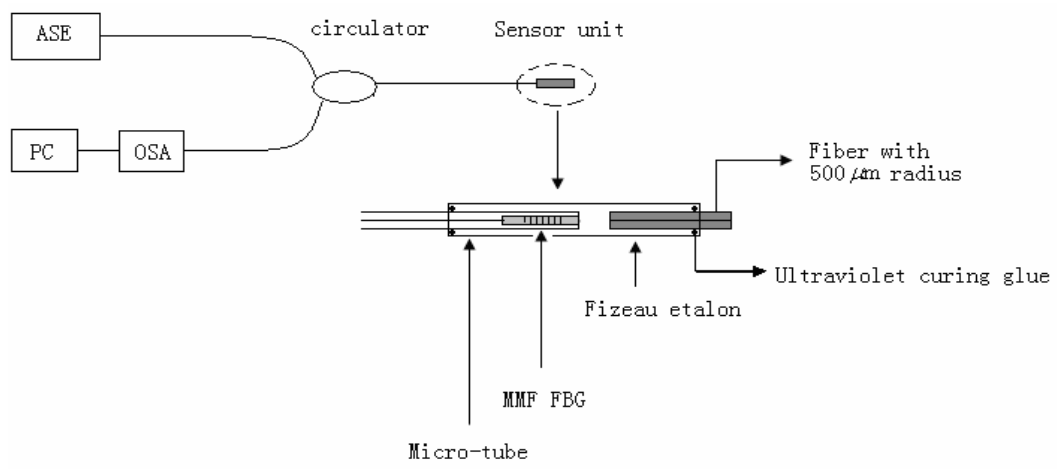

Figure 1. A GI MMF based sensor unit and its demodulation scheme.

\footnotetext{
"Corresponding author.
} 
A signal from the sensor unit is shown in Figure 2. Interference signal spectrum under different Fizeau cavity length is shown in Figure 3(a).We can use the tracking bimodal techniques to demodulate the Fizeau cavitybased sensor. We test the Fizeau cavity length by using a movable stage with $20 \mu \mathrm{m}$ resolution and the tracking bimodal method. There is a good linear relationship between the stage shift and the length of Fizeau cavity by the tracking bimodal method, shown in Figure 3(b). This proves the feasibility of the demodulation scheme for the Fizeau cavity length, and the Fizeau cavity length can be increased to $5000 \mu \mathrm{m}$ because of GI MMF used here. It is well known that the Fizeau cavity-based sensor has low crosstalk with temperature because of the minimum cavity length.

The GI MMF-Bragg grating based sensor is free from strain because of the packaging method shown in Figure 1. Its spectrum, shown in Figure 4(a), can be used to monitor environmental temperature based on the shift of the wavelength. It has 3 main reflection peaks and their temperature responses are $\sim 18.4 \mathrm{pm} / \mathrm{C}, \sim 18 \mathrm{pm} / \mathrm{C}, \sim 18.3 \mathrm{pm} / \mathrm{C}$ respectively, shown in Figure 4(b). We also test its response to curvature. By sticking this structure on a flexible steel bar and increasing the curvature of the steel bar, we found that the center wavelengths of the main peak almost do not shift, shown in Figure 4(c). However, their intensities are changed, shown in Figure 4(d), and the intensity differential between the different peaks could be used as a sensing demodulation scheme, which immune to the power vibration of light sources. So we can use GI MMF- Bragg grating measures temperature and curvature based on the wavelength shift and the intensity change, respectively.

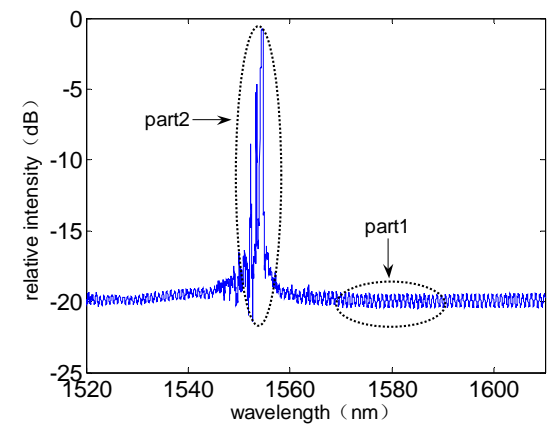

Figure 2. A signal of the GI MMF based sensor unit.
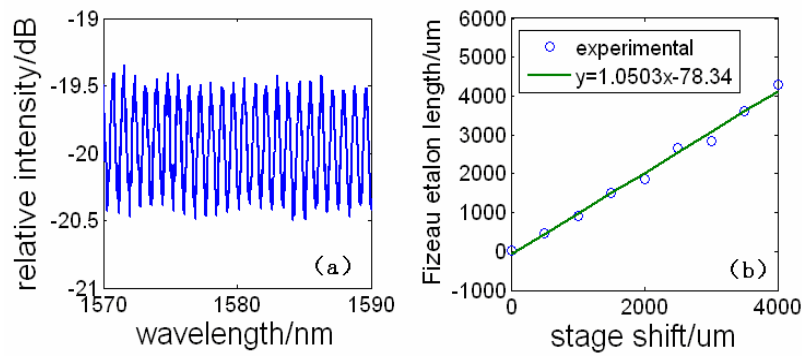

Figure 3. (a) Interference signal spectrum(part 1 in Figure 1), (b) Relationship between the stage shift and the length of Fizeau cavity.
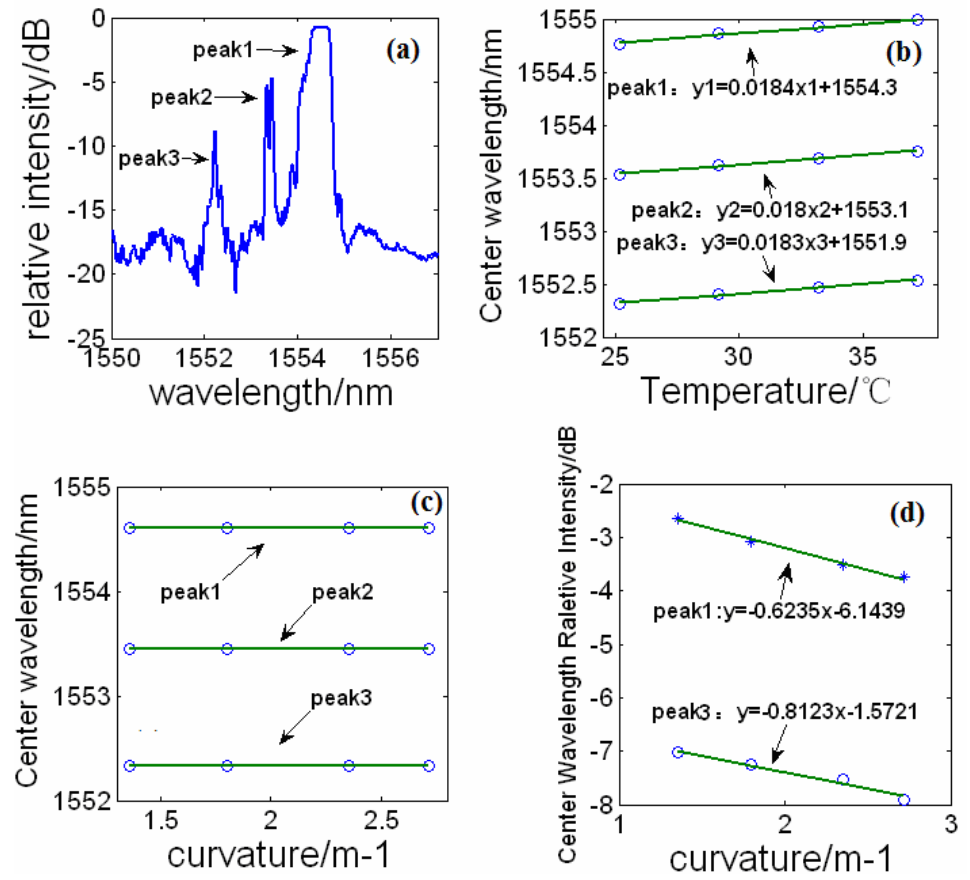

Figure 4. (a) The signal of MMF-FBG (part 2 in Figure 2), (b) The temperature response of the MMF-FBG, (c)center wavelength with the change of curvature, (d) the curvature response of the GI MMF-FBG. 
The whole measurement proves that the GI MMF Bragg grating based temperature and curvature sensor can be used to monitor the temperature individually without being affected by the applied strain, and the Fizeau cavity based sensor can simply give the strain value when reducing the thermal expansion effect of monitored structures.

\section{Discussion and Conclusions}

A single mode fiber lead-in based GI MMF Bragg grating show the different characteristics, comparing with normal Bragg gratings, and could be used to distinguish the temperature and curvature based on its wavelength and intensity. The GI MMF based Fizeau cavity is used as strain or displacement sensing by reading the interference fringes. The GI MMF based sensor unit can be multiplexed readily based on a 2 by $\mathrm{N}$ coupler. The GI MMF Bragg gratings could be distinguished by their wavelengths. The Fizeau cavity with different lengths could be discriminated by the Fast Fourier transform of the Fizeau interference spectra. We expect to multiplex much more sensor units because the long cavity range $(\sim 5 \mathrm{~mm})$ could be realized based on GI MMF fiber comparing with the single mode fiber based Fizeau sensor. We are trying to build a system for a civil engineering application and further detail experiments are going on.

In conclusion, we demonstrate a GI MMF based sensor unit to realize a multi-parameter sensing, including temperature, curvature and strain or displacement, which expect to have applications in the civil engineering area.

\section{Acknowledgements}

Authors thank for the support by National Science foundation projects $(60907034,61077063,11178010$ and LBH-Z10195), China Postdoctoral Science Foundation funded project (20100480965), Harbin Science founda- tion (2011RFLXG004) and the Fundamental Research Funds of the Central University, China.

\section{REFERENCES}

[1] Y. J. Rao, M. R. Cooper, D. A. Jackson, C. N. Pannell and L. Reekie "Simultaneous Measurement of DisplacementandTemperatureUsingIn-Fibre-Bragg-Grating-Based Extrinsic Fizeau Sensor," Electronics Letters, Vol. 36, 2000, pp. 1610-1612. doi:10.1049/el:20001134

[2] A C. L. Wong, Paul A. Childs and G. D. Peng, "Simultaneous Demodulation Technique for a Multiplexed Fiber Fizeau Interferometer and Fiber Bragg Grating Sensor System," Optics Letters, Vol. 31, 2006, pp. 23-25. doi:10.1364/OL.31.000023

[3] D. Zhou, L. Wei, W. K. Liu, Y. Liu and J. W. Y. Lit, "Simultaneous Measurement for Strain and Temperature Using Fiber Bragg Gratings and Multimode Fibers," Applied Optics, Vol. 47, 2008, pp. 1668-1672. doi:10.1364/AO.47.001668

[4] Mohammed W S, Mehta A and Johnson E G "Wavelength Tunable Fiber Lens Based on Multimode Interference," Journal of Lightwave Technology, Vol. 22, 2004, pp. 469-477. doi:10.1109/JLT.2004.824379

[5] E. Li, X. Wang and C. Zhang, "Fiber-Optic Temperature Sensor Based on Interference of Selective Higher-Order Modes," Applied Physics Letters, Vol. 89, No. 9, 2006, pp. 091119-091121. doi:10.1063/1.2344835

[6] Q.Wang, G. Farrell and W. Yan, "Investigation on Single-Mode-Multimode-Single-Mode Fiber Structure," Journal of Lightwave Technology, Vol. 26, No. 5, 2008, pp. 512-519.

[7] S. J. Peng, A. N. Zhang, J. Z. Zhang, W.M. Sun and L. B. Yuan, "SMS FBG and Its Multi-Parameters Sensing," Application Proceedings of SPIE - The International Society for Optical Engineering, 2010, p. 7853. 